\title{
Impact of Lorentz violation in the photon sector on extensive air showers
}

\author{
Frans R. Klinkhamer ${ }^{a}$, Marcus Niechciol ${ }^{*}{ }^{\text {, Markus Risse }}{ }^{b}$ \\ ${ }^{a}$ Institut für Theoretische Physik, Karlsruher Institut für Technologie (KIT), 76128 Karlsruhe, \\ Germany \\ ${ }^{b}$ Department Physik, Universität Siegen, 57068 Siegen, Germany
}

\begin{abstract}
Due to their extremely high particle energies (up to $10^{20} \mathrm{eV}$ ), cosmic rays are ideally suited to search for violations of Lorentz invariance. We consider isotropic, nonbirefringent Lorentz violation in the photon sector and specialize to the case of a photon velocity larger than the maximum attainable velocity of standard Dirac fermions. Up to now, bounds on this type of Lorentz violation have been determined from observations of $\mathrm{TeV}$ gamma rays.

In this contribution, we present a novel approach to test Lorentz invariance with improved sensitivity. This approach is based on the measurement of extensive air showers which are induced by cosmic-ray particles in the Earth's atmosphere. We study the impact of Lorentz-violating processes (e.g. photon decay into an electron-positron pair) on the longitudinal development of air showers, in particular on the atmospheric depth of the shower maximum $X_{\max }$. We use Monte Carlo simulations performed with the CONEX simulation code which was extended to include Lorentz-violating processes at a magnitude still allowed by present bounds. We show that taking into account these processes reduces the average $X_{\max }$ for showers with primary energies above $10^{18} \mathrm{eV}$ compared to standard physics by an amount that is much higher than the average resolution of current air shower experiments.
\end{abstract}

35th International Cosmic Ray Conference - ICRC2017

10-20 July, 2017

Bexco, Busan, Korea

* Speaker. 


\section{Introduction}

Ever since its inception, the Standard Model of Elementary Particle Physics (SM) has been extremely successful, with its predictions being tested to very high precision. However, it is well known that the SM is not complete, as it, e.g., describes neither gravity nor dark matter. Current approaches to complete the picture and establish a more fundamental theory allow for deviations from exact Lorentz symmetry. The determination of some of the best current limits on Lorentz violation (LV) in various sectors of the SM has taken advantage of the high energies of cosmic rays and gamma rays $[1,2,3]$.

We study the impact of LV on extensive air showers initiated by ultra-high-energy (UHE) cosmic rays with energies above $1 \mathrm{EeV}=10^{18} \mathrm{eV}$ in the Earth's atmosphere. In particular, we consider isotropic, nonbirefringent LV in the photon sector, specializing to the case of a photon velocity larger than the maximum attainable velocity of standard Dirac fermions. This approach was first studied in [4], where an analytical ansatz was used, modifying the well-known Heitler model for electromagnetic cascades to include LV through photon decay. A significant impact on the longitudinal shower development was found. Here, we build upon that previous work, employing a full Monte Carlo (MC) ansatz, which allows us to study in detail the impact of LV not only on pure electromagnetic cascades, but also on air showers initiated by hadrons. In addition to the decay of secondary photons with energies above the LV threshold in the air shower, also a related modification of the decay of neutral pions has to be accounted for.

The theory background of LV in the context of our study is briefly summarized in Sec. 2. The results of our MC study are presented in Sec. 3, in particular the changes in the average atmospheric depth of the shower maximum $\left\langle X_{\max }\right\rangle$ due to $L V$ for pure electromagnetic cascades (for a comparison with [4]) and for hadron-induced air showers. In addition, we present improved an bound on this type of LV through a comparison of our results with $\left\langle X_{\max }\right\rangle$ measurements (Sec. 3.3).

\section{Theory}

In a relatively simple extension of standard quantum electrodynamics (QED), a single term which breaks Lorentz invariance but preserves CPT and gauge invariance $[5,6]$ is added to the Lagrange density ${ }^{1}$ :

$$
\mathscr{L}=\underbrace{-\frac{1}{4} F^{\mu v} F_{\mu v}+\bar{\psi}\left[\gamma^{\mu}\left(i \partial_{\mu}-e A_{\mu}\right)-m\right] \psi}_{\text {standard QED }}-\underbrace{\frac{1}{4}\left(k_{F}\right)_{\mu v \rho \sigma} F^{\mu v} F^{\rho \sigma}}_{\text {CPT-even LV term }} .
$$

The constant fixed "tensor" $\left(k_{F}\right)_{\mu v \rho \sigma}$ has 20 independent components, ten of which produce birefringence and eight of which lead to direction-dependent modifications of the photon propagation. The remaining two components correspond to an isotropic modification of the photon propagation and an unobservable double trace that changes the normalization of the photon field. Isotropic, nonbirefringent $\mathrm{LV}$ in the photon sector is then controlled by a single dimensionless parameter $\kappa$,

\footnotetext{
${ }^{1}$ Note that the Minkowski metric $g_{\mu \nu}(x)=\eta_{\mu \nu}=[\operatorname{diag}(+1,-1,-1,-1)]_{\mu \nu}$ and natural units $(\hbar=c=1)$ are used throughout this article.
} 
which enters the fixed tensor $k_{F}$ in Eq. 2.1 in the following way:

$$
\left(k_{F}\right)_{\mu \lambda v}^{\lambda}=\frac{\kappa}{2}[\operatorname{diag}(3,1,1,1)]_{\mu v} .
$$

In physical terms, the velocity $c$ corresponds to the maximum attainable velocity of the massive Dirac fermion in Eq. 2.1, whereas the phase velocity of the photon is given by

$$
v_{\mathrm{ph}}=\frac{\omega}{|\vec{k}|}=\sqrt{\frac{1-\kappa}{1+\kappa}} c,
$$

which is smaller (larger) than $c$ for positive (negative) values of $\kappa$.

For non-zero values of $\kappa$, certain processes that are forbidden in the conventional, Lorentzinvariant theory become allowed. We focus here on the case $\kappa<0$, where photons become unstable above the energy threshold

$$
E_{\gamma}^{\mathrm{th}}(\kappa)=2 m_{e} \sqrt{\frac{1-\kappa}{-2 \kappa}} \simeq \frac{2 m_{e}}{\sqrt{-2 \kappa}}
$$

with $m_{e}=511 \mathrm{keV}$ the rest mass of the electron. Photons above this threshold decay very efficiently into electron-positron pairs. At tree level, the decay rate of the photon decay $(\mathrm{PhD})$ as a function of the photon energy $E_{\gamma} \geq E_{\gamma}^{\text {th }}$ is given by [3,7]

$$
\Gamma_{\mathrm{PhD}}\left(E_{\gamma}\right)=\frac{\alpha}{3} \frac{-\kappa}{1-\kappa^{2}} \sqrt{E_{\gamma}^{2}-\left(E_{\gamma}^{\mathrm{th}}\right)^{2}}\left(2+\left(E_{\gamma}^{\mathrm{th}}\right)^{2} / E_{\gamma}^{2}\right),
$$

with $E_{\gamma}^{\text {th }}$ from Eq. 2.4 and the fine-structure constant $\alpha \equiv e^{2} /(4 \pi) \approx 1 / 137$. Only below-threshold photons from astrophysical sources will reach Earth. Therefore, terrestrial observations of gamma rays with energies of the order $100 \mathrm{TeV}$ from distant sources using ground-based Cherenkov telescopes can be used to impose limits on $\kappa<0$, with the most stringent current limit being $\kappa>$ $-9 \times 10^{-16}$ at a confidence level (C.L.) of $98 \%$ [3].

To improve this limit on $\kappa<0$, higher-energy photons than currently observed would be needed. So far, despite extensive searches for astrophysical (primary) photons with $\mathrm{PeV}$ or EeV energies, no unambiguous photon detection could be reported at these energies (see e.g. [8]). However, photons with energies $\gg 100 \mathrm{TeV}$ are expected to be produced as secondary particles when a UHE hadron enters the Earth's atmosphere and initiates an air shower. In the first interaction of the primary hadron with an atmospheric nucleus, mostly charged and neutral pions are produced. The charged pions further interact with particles from the atmosphere, producing more pions, while the neutral pions rapidly decay into pairs of photons, which in turn trigger electromagnetic subshowers. Especially in the start-up phase of the air shower, where the energy of the secondary particles is very high, a modification of the particles due to LV (e.g. the immediate decay of abovethreshold photons) can drastically modify the overall development of the air shower, as shown in [4] for electromagnetic cascades.

Since we want to study air showers induced by hadrons, we also have to take into account possible related modifications of other processes due to LV, which may also have an influence on the development of the air shower. The relevant process here is the decay of the neutral pion into two photons: in the context of the theory considered here, the decay time $\tau$ of the neutral pion is modified by a factor depending on the energy $E_{\pi^{0}}$ of the pion and $\kappa$ [9]:

$$
\tau\left(E_{\pi^{0}}, \kappa\right)=\frac{\tau_{\mathrm{SM}}}{g\left(E_{\pi^{0}}, \kappa\right)},
$$


with $\tau_{\mathrm{SM}}$ denoting the decay time in the conventional, Lorentz-invariant theory and the factor $g\left(E_{\pi^{0}}, \kappa\right)$ given by

$$
g\left(E_{\pi^{0}}, \kappa\right)= \begin{cases}\frac{\sqrt{1-\kappa^{2}}}{(1-\kappa)^{3}}\left[1-\left(E_{\pi^{0}} / E_{\pi^{0}}^{\mathrm{th}}\right)^{2}\right]^{2} & \text { for } E_{\pi^{0}}<E_{\pi^{0}}^{\mathrm{th}}, \\ 0 & \text { otherwise. }\end{cases}
$$

The pion threshold parameter $E_{\pi^{0}}^{\text {th }}$ in Eq. 2.7 is given by

$$
E_{\pi^{0}}^{\mathrm{th}}=m_{\pi^{0}} \sqrt{\frac{1+\kappa}{-2 \kappa}} \simeq \frac{m_{\pi^{0}}}{\sqrt{-2 \kappa}} \simeq \frac{m_{\pi^{0}}}{2 m_{e}} E_{\gamma}^{\text {th }} \simeq 132 E_{\gamma}^{\text {th }},
$$

using Eq. 2.4 and $m_{\pi^{0}}=134.98 \mathrm{MeV}$ denoting the rest mass of the neutral pion. Thus, the neutral pions start to be modified at energies about two orders of magnitude above the photon threshold.

\section{Simulation Study}

In the following, we discuss the results of our LV study using a full MC approach. Since we are mainly interested in the changes in the longitudinal development of air showers, we use the MC code CONEX v2r5p40 [10,11], which we extended to include the decay of photons as well as the modified decay time of neutral pions. Photon decay is implemented as the immediate decay of photons above the threshold given by Eq. 2.4 into electron-positron pairs, with the energy distributions of the secondary particles modeled according to [7]. For the modified decay time of neutral pions Eqs. 2.6-2.8 have been implemented into the CONEX code.

To describe hadronic interactions in the MC simulation of the air showers, we use the EPOS LHC model [12]. For all other settings, we use the defaults provided by the CONEX code.

\subsection{Primary photons}

To compare the results of the full MC simulation with those of the analytical Heitler-type model of [4], we first consider the case of primary photons. In Fig. 1, the average atmospheric depth of the shower maximum $\left\langle X_{\max }\right\rangle$ is shown as a function of the primary energy for different values of $\kappa$. The significant reduction of $\left\langle X_{\max }\right\rangle$ and the change of elongation rate is confirmed; for instance, at $10^{19} \mathrm{eV},\left\langle X_{\max }\right\rangle$ can be reduced by up to $320 \mathrm{~g} \mathrm{~cm}^{-2}$. The full simulation shows a larger decrease compared to the Heitler model. This can be understood: the previous analytical approach neglected that showers initiated by electrons/positrons are shorter than those initiated by photons of same energy, and LV here converts the primary photon directly into an electron-positron pair. A further difference comes from using realistic energy distributions (compared to the equal energy sharing assumed previously).

\subsection{Primary hadrons}

We now regard the case of secondary photons produced in air showers initiated by primary hadrons. This has not been studied before and requires an account of the modification of the neutral pion decay as well (see Sec. 2). As can be seen in Fig. 2 (left) for primary protons and iron nuclei, also hadron-induced air showers are significantly affected in terms of both $\left\langle X_{\max }\right\rangle$, which is up to 


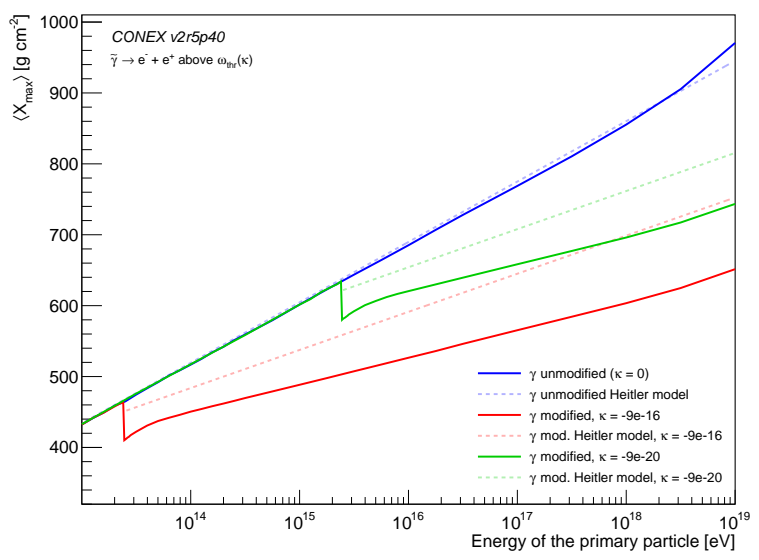

Figure 1: Average atmospheric depth of the shower maximum $\left\langle X_{\max }\right\rangle$ as a function of the primary energy for primary photons, taken from MC simulations perfomed with the CONEX code which was modified to include LV. The dashed lines indicate the $\left\langle X_{\max }\right\rangle$ expected from the analytical Heitler-type model of [4].
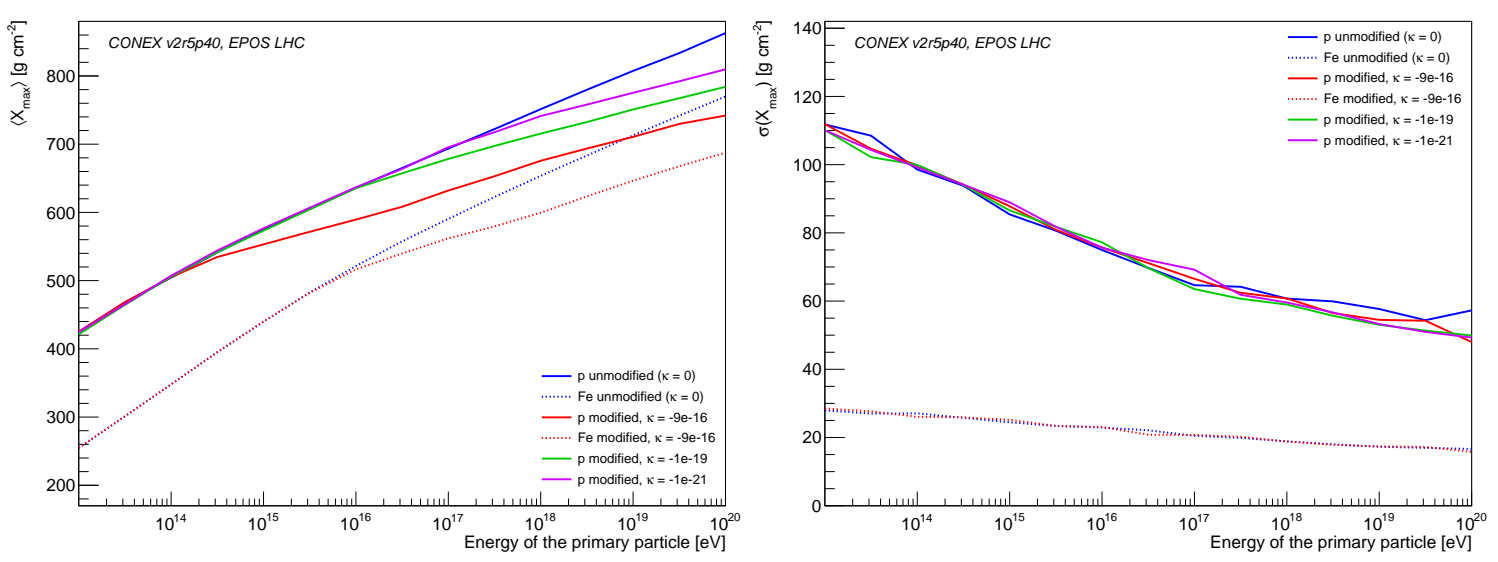

Figure 2: Left: $\left\langle X_{\max }\right\rangle$ as a function of the primary energy for primary protons and iron nuclei; right: $\sigma\left(X_{\max }\right)$ as a function of the primary energy for primary protons and iron nuclei.

$100 \mathrm{~g} \mathrm{~cm}^{-2}$ smaller than in the unmodified case for protons at $10^{19} \mathrm{eV}$, and elongation rate, which is reduced by about $20 \mathrm{~g} \mathrm{~cm}^{-2}$ per decade for protons at energies above $10^{18} \mathrm{eV}$, independent from $\kappa$. The modification of $\left\langle X_{\max }\right\rangle$ starts when the energy per nucleon of the primary particle exceeds the LV threshold energy by a factor $\sim 10$, since then secondary photons above threshold are produced. The onset of neutral pions becoming stable occurs about two orders of magnitude higher in energy (cf. Eq. 2.8). It is noticed as a small effect only, i.e. a slight upturn of the modified curve. The curves are shifted as expected with respect to each other for changed values of $\kappa$ and when primary iron nuclei are used instead of protons (this corresponds to a factor 56 smaller energy per nucleon).

The impact of the LV modification on the $X_{\max }$ fluctuations can be studied as well. Interestingly, while $\left\langle X_{\max }\right\rangle$ is significantly affected, the modification leaves $\sigma\left(X_{\max }\right)$ unchanged, see Fig. 2 (right). This is understandable, as the shower-to-shower fluctuations are dominated by fluctuations in the first interactions of the highest-energy hadronic primaries, which remain unmodified here.

Using different primary nuclei (proton, Helium, Oxygen, Iron), we determined the values of $\left\langle X_{\max }\right\rangle$ as a function of $\kappa$ at a fixed primary energy of $10^{19} \mathrm{eV}$, see Fig. 3. $\left\langle X_{\max }\right\rangle$ scales linearly with 


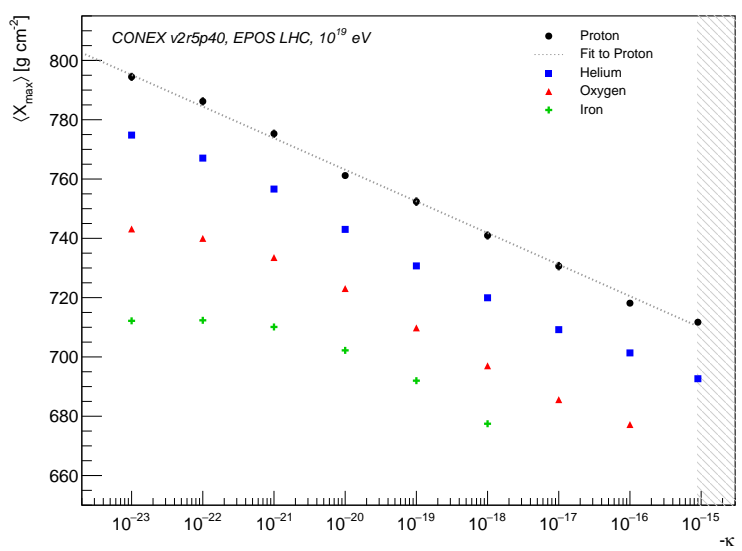

Figure 3: $\left\langle X_{\max }\right\rangle$ as a function of $-\kappa$ for different primary nuclei (proton, Helium, Oxygen and Iron) a fixed primary energy of $10^{19} \mathrm{eV}$. The dotted line denotes a linear fit to the proton distribution, while the hatched area indicates the range of $-\kappa$ values that are excluded by previous bounds [3].

$\log _{10}(-\kappa)$, if the threshold energy is well below the energy per nucleon of the primary particle. This can be used to obtain a parametrization of $\left\langle X_{\max }\right\rangle$ as a function of the primary energy $E$, the primary mass $A$ (here, we consider only the proton case $A=1$ ) and the $\operatorname{LV}$ parameter $\kappa<0$. For EPOS LHC, this parameterization is given by

$$
\left\langle X_{\max }\right\rangle(E, A=1, \kappa)=p_{0}+p_{1} \log _{10}(-\kappa)+p_{2}\left(\log _{10}(E[\mathrm{eV}])-19\right),
$$

where $p_{0}=(550 \pm 2) \mathrm{g} \mathrm{cm}^{-2}$ and $p_{1}=(-10.7 \pm 0.1) \mathrm{g} \mathrm{cm}^{-2}$ are determined through a fit to the proton distribution shown in Fig. 3 and $p_{2}=(34.3 \pm 0.3) \mathrm{g} \mathrm{cm}^{-2}$ is the $\kappa$-independent elongation rate taken from Fig. 2 (left).

We also checked the dependence of the simulations on the choice of the hadronic interaction model, using QGSJET-II-04 [15] and SIBYLL 2.3 [13, 14] instead of EPOS LHC. As expected, the LV-modified curves reflect the differences between the unmodified ones for the different interaction models. Differences between the models of the modified $\left\langle X_{\max }\right\rangle$ values are smaller compared to the unmodified $\left\langle X_{\max }\right\rangle$ values due to the reduced elongation rates. For proton primaries, the differences in $\left\langle X_{\max }\right\rangle$ are about $10-15 \mathrm{~g} \mathrm{~cm}^{-2}$ compared to EPOS LHC, with QGSJET-II-04 (SIBYLL 2.3) giving smaller (larger) $\left\langle X_{\max }\right\rangle$ values. These differences are small compared to the overall reduction of $\left\langle X_{\max }\right\rangle$ by the $\mathrm{LV}$ modification allowed by present bounds.

\subsection{Comparison to $\left\langle X_{\max }\right\rangle$ measurements}

A comparison of our simulations with $\left\langle X_{\max }\right\rangle$ measurements is given in Fig. 4. Only measurements from the Pierre Auger Observatory [16] are shown here since the $\left\langle X_{\max }\right\rangle$ measurements from the Telescope Array (TA) are not corrected for detector effects and since TA measurements were found to be consistent with Auger measurements [17]. For the maximum LV allowed by previous work $\left(\kappa=-9 \times 10^{-16}[3]\right)$, it can be seen that $\left\langle X_{\max }\right\rangle$ predictions are significantly below the observations, irregardless of any assumptions on the primary mass and the interaction model. Thus, stricter constraints than before can be placed on $\kappa$ by this method.

Allowing most conservatively for the case of a pure proton composition, one can see that also the case of $\kappa=-10^{-19}$ is still below the observed values above $10^{18} \mathrm{eV}$. Focusing on energies 


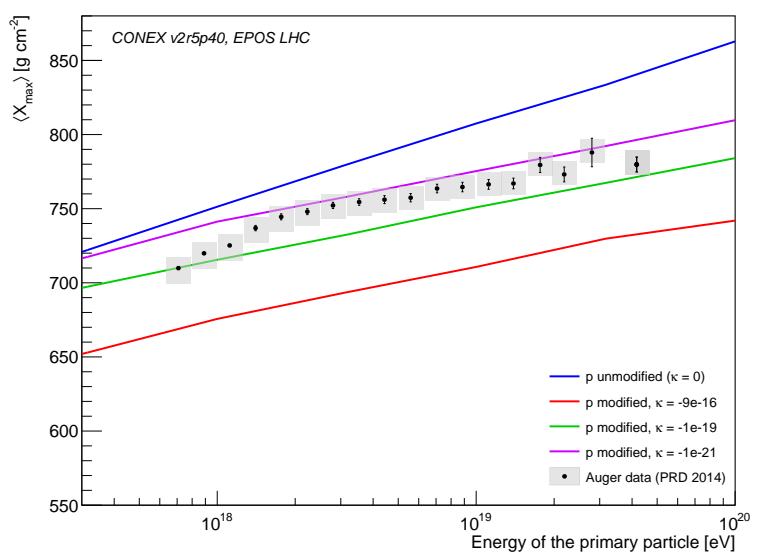

Figure 4: $\left\langle X_{\max }\right\rangle$ as a function of the primary energy for primary protons compared to $\left\langle X_{\max }\right\rangle$ measurements from the Pierre Auger Observatory [16]. The gray boxes around the data points indicate the systematic uncertainties of the measurements.

around $3 \times 10^{18} \mathrm{eV}$ and taking the uncertainties on the measured $\left\langle X_{\max }\right\rangle$ values into account, a preliminary improved limit of $\kappa=-1.4 \times 10^{-19}$ (at $98 \%$ C.L.) can be determined. This limit is based on simulations with EPOS LHC (using Eq. 3.1) where a systematic uncertainty of $15 \mathrm{~g} \mathrm{~cm}^{-2}$ has been assumed to account for the uncertainties related to the choice of the hadronic interaction model. The limit on $\kappa<0$ determined here improves the previous bound by a factor $\sim 6400$. As a comparison to the previous approach from [3], the observation of a primary photon with an energy of about $2 \mathrm{PeV}$ would be needed to get the same improvement of the bound on $\kappa<0$.

\section{Summary and discussion}

By implementing Lorentz-violating effects (photon decay and the related suppression of the neutral pion decay) in a full MC shower simulation, we studied the impact of LV on air showers initiated by UHE cosmic rays. This method exploits the expected production of secondary photons with energies $\gg 100 \mathrm{TeV}$, and the accelerated shower development due to photon decay. The average depth of the shower maximum $\left\langle X_{\max }\right\rangle$ is reduced by up to $100 \mathrm{~g} \mathrm{~cm}^{-2}$ at $10^{19} \mathrm{eV}$, with the difference increasing with energy due to a reduced elongation rate. This is well above the typical $X_{\max }$ resolution of current air shower experiments. For example, the $X_{\max }$ resolution of the Pierre Auger Observatory is better than $26 \mathrm{~g} \mathrm{~cm}^{-2}$ at energies around $10^{17.8} \mathrm{eV}$, improving with energy to about $15 \mathrm{~g} \mathrm{~cm}^{-2}$ above $10^{19.3} \mathrm{eV}$ [16]. The shower fluctuations $\sigma\left(X_{\max }\right)$ are not affected by the LV modification. While not the focus of the present work, we also started to check the muon component of the air shower (see e.g. [18] for considerations of shower muons and LV). As an initial result, the number of muons on ground level was found to increase significantly above the threshold energy for the suppression of the neutral pion decay.

For a value of $\kappa=-9 \times 10^{-16}$ as allowed so far by the previous bound, the predictions of $\left\langle X_{\max }\right\rangle$ are at odds with measurements, irrespective of the primary mass and the interaction model: much deeper showers are observed than expected. From $\left\langle X_{\max }\right\rangle$ alone, a preliminary improved bound of $\kappa=-1.4 \times 10^{-19}$ (at $98 \%$ C.L.) is estimated. For this new bound, the primary compo- 
sition has most conservatively been assumed to consist of protons only. Heavier primaries have smaller $\left\langle X_{\max }\right\rangle$ values, which would lead to stronger bounds.

In fact, a pure proton composition above $3 \times 10^{18} \mathrm{eV}$ can be regarded to be excluded already by air shower measurements. Specifically, observations of $\sigma\left(X_{\max }\right)[16]$ and of the correlation between $X_{\max }$ and the ground signal [19] show a mixed composition with a significant fraction of heavier nuclei. Taking this into account will provide further improvements of the bound on $\kappa$. As an illustration, let us assume that the average primary mass is $\langle A\rangle \simeq 4$. Then, the resulting predicted $\left\langle X_{\max }\right\rangle$ would coincide with that of primary Helium. For a given observed value of $\left\langle X_{\max }\right\rangle$, this would imply a factor $\sim 100$ improvement on the bound on $\kappa$ according to Fig. 3. We leave this analysis for future work.

\section{Acknowledgments}

We thank T. Pierog for his help in modifying the CONEX source code. In addition, we kindly acknowledge helpful discussions with J. S. Díaz. This work was partially funded by the German Research Foundation (DFG), the Federal Ministry of Education and Research (BMBF), and the Helmholtz Alliance for Astroparticle Physics (HAP).

\section{References}

[1] F. R. Klinkhamer and M. Risse, Phys. Rev. D 77 (2008) 016002.

[2] F. R. Klinkhamer and M. Risse, Phys. Rev. D 77 (2008) 117901.

[3] F. R. Klinkhamer and M. Schreck, Phys. Rev. D 78 (2008) 085026.

[4] J. S. Díaz, F. R. Klinkhamer and M. Risse, Phys. Rev. D 94 (2016) 085025.

[5] S. Chadha and H. B. Nielsen, Nucl. Phys. B 217 (1983) 124.

[6] V. A. Kostelecký and M. Mewes, Phys. Rev. D 66 (2002) 056005.

[7] J. S. Díaz and F. R. Klinkhamer, Phys. Rev. D 92 (2015) 025007.

[8] M. Niechciol for the Pierre Auger Collaboration, these proceedings.

[9] F. R. Klinkhamer, arXiv:1610.03315.

[10] T. Bergmann, et al., Astropart. Phys. 26 (2007) 420.

[11] T. Pierog, et al., Nucl. Phys. Proc. Suppl. 151 (2006) 159.

[12] T. Pierog, et al., Phys. Rev. C 92 (2015) 034906.

[13] E.-J. Ahn, et al., Phys. Rev. D 80 (2009) 094003.

[14] F. Riehn, et al., PoS(ICRC2015) 558.

[15] S. Ostapchenko, Phys. Rev. D 83 (2011) 014018.

[16] The Pierre Auger Collaboration, Phys. Rev. D 90 (2014) 122005.

[17] M. Unger for the Pierre Auger and Telescope Array Collaborations, PoS(ICRC2015) 307.

[18] G. Tomar, Phys. Rev. D 95 (2017) 095035.

[19] The Pierre Auger Collaboration, Phys. Lett. B 762 (2016) 288. 